\title{
Alternatively spliced BobCAL transcripts alter curd morphotypes in a collection of Chinese cauliflower accessions
}

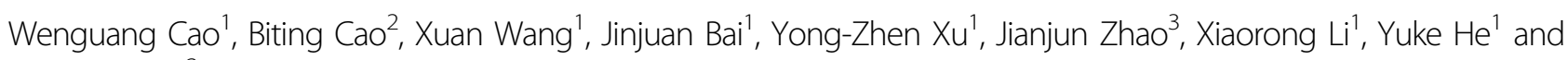
Shengwu $\mathrm{Hu}^{2}$

\begin{abstract}
The curd of cauliflower (Brassica oleracea L. var. botrytis) is a modified inflorescence that is consumed as a vegetable. Curd formation is proposed to be due to a mutation in the BobCAULIFLOWER (BobCAL) gene, but the genetic relationship between BobCAL variation and curd morphotypes remains obscure. To address this question, we collected and classified a collection of 78 cauliflower accessions into four subpopulations according to curd surface features: smooth, coarse, granular, and hairy curd morphotypes. Through the cDNA sequencing of BobCAL alleles, we showed that smooth and coarse accessions characterized by inflorescence meristem arrest presented a strong association with the 451T SNP (BobCAL_T), whereas granular and hairy accessions marked with floral organ arrest presented an association with 451G (BobCAL_G). Interestingly, all BobCAL alleles were alternatively spliced, resulting in a total of four alternative splice (AS) variants due to the retention of the fourth and/or seventh introns. Among accessions with BobCAL_G alleles, the total expression of all these AS variants in granular plants was almost equal to that in hairy plants; however, the expression of the individual AS variants encoding intact proteins relative to those encoding truncated proteins differed. Hairy accessions showed relatively high expression of the individual variants encoding intact proteins, whereas granular accessions displayed relatively low expression. In smooth cauliflower, the overexpression of the BobCAL_Ga variant caused an alteration in the curd morphotype from smooth to hairy, concurrent with an increase in the expression levels of downstream floral identity genes. These results reveal that alternative splicing of BobCAL transcripts is involved in the determination of cauliflower curd morphotypes.
\end{abstract}

\section{Introduction}

The species Brassica oleracea includes several cultivated subspecies with extreme morphological divergence of various organs due to the proliferation of different types of meristems. Among these subspecies, cabbage (B. oleracea L. var. capitata), kale (B. oleracea L. var. acephala),

\footnotetext{
Correspondence: Xiaorong Li (xrli@sippe.ac.cn) or Yuke He (ykhe@sibs.ac.cn) or Shengwu Hu (swhu83251@snwafu.edu.cn)

'National Laboratory of Plant Molecular Genetics, Center for Excellence in Molecular Plant Sciences, Shanghai Institutes of Plant Physiology \& Ecology, Chinese Academy of Science, 300 Fenglin Road, Shanghai 200032, China ${ }^{2}$ State Key Laboratory of Crop Stress Biology in Arid Areas and College of Agronomy, Northwest A\&F University, Yangling, Shaanxi, China Full list of author information is available at the end of the article These authors contributed equally: Wenguang Cao, Biting Cao
}

and Brussels sprouts ( $B$. oleracea L. var. gemmifera) exhibit elongated inflorescences, while cauliflower $(B$. oleracea L. var. botrytis) and broccoli (B. oleracea L. var. italica) are characterized by inflorescences that are modified into large dense structures (curds). These varying forms are due to selection for different characteristics during domestication ${ }^{1}$. Cauliflower curd is one of the most important vegetable products. Curd induction and development have been previously investigated by a number of researchers (for reviews ${ }^{1,2}$ ). Although curd formation was originally considered to be a vegetative process $^{3-6}$, the typical curd is now considered to be a dense mass of stage-arrested inflorescence/floral meristems derived from their own iterative proliferation ${ }^{7,8}$.

\section{(c) The Author(s) 2020}

(c) (i) Open Access This article is licensed under a Creative Commons Attribution 4.0 International License, which permits use, sharing, adaptation, distribution and reproduction c. in any medium or format, as long as you give appropriate credit to the original author(s) and the source, provide a link to the Creative Commons license, and indicate if changes were made. The images or other third party material in this article are included in the article's Creative Commons license, unless indicated otherwise in a credit line to the material. If material is not included in the article's Creative Commons license and your intended use is not permitted by statutory regulation or exceeds the permitted use, you will need to obtain permission directly from the copyright holder. To view a copy of this license, visit http://creativecommons.org/licenses/by/4.0/. 
At the curd formation stage, the extension of the inner inflorescence branches is restricted, while the inflorescence/floral meristems on the surface temporarily lose the ability to generate floral organs and instead repeatedly copy themselves. This arrest of the floral organ generation ability persists until external conditions, such as temperature and day length, and internal cues are appropriate for continued floral development. The exploration of the mechanisms controlling the cauliflower curd phenotype can contribute to the improvement of product quality and aid in the understanding of the general control of floral development. In regard to this goal, little progress had been achieved until the CAULIFLOWER phenotype, resembling the cauliflower curd, was identified and characterized in the ap1 cal mutant of Arabidopsis thaliana, a close relative of cauliflower ${ }^{9,10}$.

In the ap1 cal double mutant, floral meristems that give rise to flowers in the wild type instead behave as inflorescence meristems, which in turn produce higher-order inflorescence meristems in a phyllotactic spiral on their flanks ${ }^{9,11}$. As a result, these plants fail to undergo the normal inflorescence-to-floral transition and only occasionally produce floral organs.

$C A L$ and $A P 1$ are closely related genes that share redundant roles in the specification of floral meristem identity $^{12}$. Both genes encode MADS-domain proteins composed of four different domains, designated as MADS $(\mathrm{M})$, intervening (I), keratin-like (K) and C-terminal (C). Studies of MADS-domain proteins in diverse species have shown that AP1 and CAL can each interact with a shared set of proteins, including SEPALLATA3 (SEP3), SOC1, SVP, and AGL24 ${ }^{13-16}$. In addition, the $M$ domain is responsible for binding to DNA. The I region participates in homodimer formation ${ }^{17,18}$. The $\mathrm{K}$ domain has been implicated in protein-protein interactions ${ }^{17-21}$ and is postulated to form several amphipathic $\alpha$-helices, referred to as $\mathrm{K} 1, \mathrm{~K} 2$, and $\mathrm{K} 3 . \mathrm{K} 2$ and $\mathrm{K} 1$ are required for many interactions, such as PI/SEP3 (or PI/SEP1) interaction ${ }^{22}$. The C-terminal region has been proposed to be involved in transcriptional activation ${ }^{23}$.

Consistent with the observed similarities between A. thaliana and cauliflower curd phenotypes, a mutation has been found in BobCAL, a $C A L$ gene ortholog in cauliflower considered to be associated with curd formation ${ }^{10}$. In a study of $B$. oleracea, a nonsense mutation in exon 5 of the BoCAL gene was shown to be nearly fixed in most accessions of cauliflower and broccoli but was also present in some accessions of cabbage and kale, which do not produce curds. The mutant allele has also been found in some broccoli and noncurding Brassica accessions ${ }^{24,25}$. On the other hand, the wild-type BobCAL allele without this nonsense mutation can occur in cauliflower ${ }^{24}$. A subsequent survey of broccoli and cauliflower accessions found only a weak association between BobCAL mutant alleles and cauliflower phenotypes ${ }^{26}$. These results suggest that the molecular mechanism underlying curd phenotypes is more complicated than expected.

To understand the genetic relationship between Bob$C A L$ and curd phenotypes, we classified a collection of cauliflower accessions and cloned the cDNAs of their BobCAL genes. We first distinguished granular and hairy curd phenotypes from this population according to curd texture and the curd surface. cDNA sequencing then revealed that some accessions with granular or hairy curd phenotypes lacked the BobCAL nonsense mutation. To our surprise, several alternative splice (AS) variants were identified from the different BobCAL alleles. More importantly, we found that differences in expression levels among these splice variants were the molecular cause of the granular and hairy curd phenotypes. This conclusion was verified by the overexpression of a BobCAL variant with normal function. We have thus determined that the AS of BobCAL alleles alters curd phenotypes.

\section{Results \\ Variation in curd morphotypes}

In this study, 78 cauliflower accessions were collected from different sources. To avoid heterogeneity, all accessions were self-fertilized for more than five generations, and each accession was represented by an inbred line. These inbred lines were grown in the field during the same seasons in 2016-2018. We measured curd-related traits such as developmental arrest, the width of curds, the number of days to curd maturity, and the number of days to flowering. The parameters of curd-related traits did not exhibit distinct differences between the years. Hence, we used the parameters recorded in all 3 years for the analysis of frequency distribution (Supplementary Fig. 1). The variance in the curd-related traits between the accessions was high. To analyze the genetic basis of their curd phenotypes, these accessions were classified according to their curd texture into two categories: tightly compact or loosely compact (Fig. 1a-d). Tightly compact curds were difficult to divide by hand, whereas those classified as loosely compact came apart easily. On the basis of curd surface features, accessions with tightly compact curds were subclassified into smooth curd $(s c)$ and coarse curd (cc) subpopulations (Fig. 1e-h), while those exhibiting loosely compact curds were divided into granular curd $(g c)$ and hairy curd $(h c)$ categories. The number of accessions in each subpopulation was 41 in $s c, 16$ in $c c, 10$ in $g c$ and 11 in $h c$ (Table 1), where $s c$ corresponds to the "classic" curd phenotype, accounting for $50 \%$ of the total accession.

We next examined the progress of curd development. At the early reproductive stage, the inflorescence meristems of smooth curds resembled broad domes from which secondary inflorescence meristems arose and 

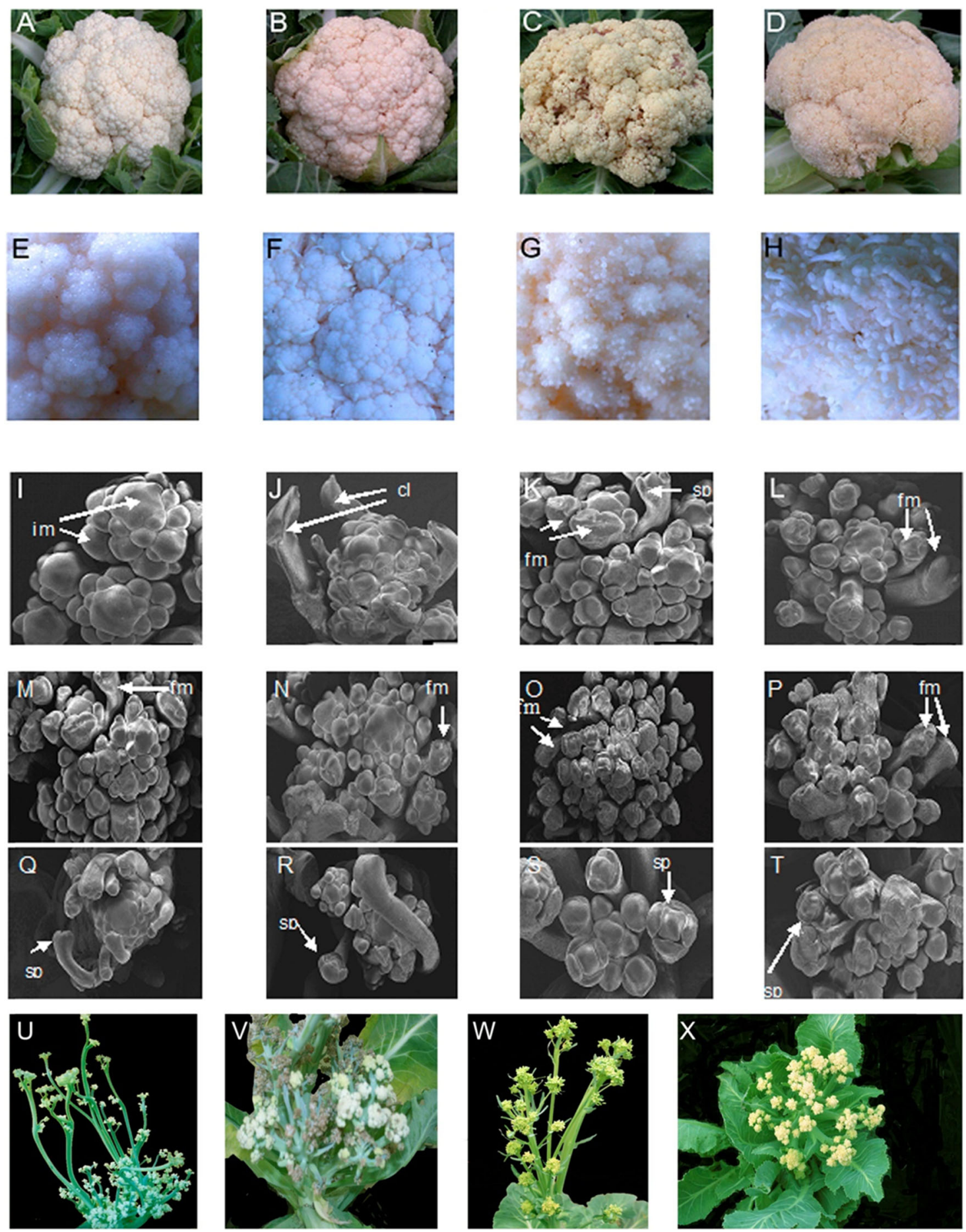

Fig. 1 Curds of four types of cauliflower accessions Morphotypes of smooth (a), coarse (b), granular (c), and hairy (d) curds of cauliflower at the curd maturation stage. Surfaces of smooth $(\mathbf{e})$, coarse $(\mathbf{f})$, granular $(\mathbf{g})$, and hairy $(\mathbf{h})$ curds. i-t Scanning electron micrographs of inflorescence, floral, and organ meristems. Inflorescence meristems of smooth (i) and coarse (j) curds and floral meristems of granular (k) and hairy (I) curds at the curd enlargement stage. Inflorescence meristems of smooth $(\mathbf{m})$ and coarse $(\mathbf{n})$ accessions and floral meristems of granular $(\mathbf{o})$ and hairy $(\mathbf{p})$ accessions at the bolting stage in curds during winter. Flower buds of smooth $(\mathbf{q})$ and coarse $(\mathbf{r})$ accessions and floral meristems of granular $(\mathbf{s})$ and hairy $(\mathbf{t})$ accessions at the flowering stage, immediately after bolting. Inflorescences of smooth ( $\mathbf{u}$ ) and coarse $(\mathbf{v})$ accessions and floral meristems of granular $(\mathbf{w})$ and hairy $(\mathbf{x})$ accessions at the bolting stage in curds during winter. Arrows indicate meristems or floral organs. im inflorescence meristems, $\mathrm{Cl}$ cauline leaves, sp sepal primordia, fm floral meristems. 
Table 1 Association of curd phenotypes with the G451T single-nucleotide polymorphism in a collection of 78 cauliflower accessions.

\begin{tabular}{llcl}
\hline Curd phenotypes & \multicolumn{2}{l}{ Number of accessions with } \\
\cline { 2 - 4 } & BobCAL_T & BobCAL_G & Sum \\
\hline Smooth & 38 & 3 & 41 \\
Coarse & 15 & 1 & 16 \\
Granular & 0 & 10 & 10 \\
Hairy & 1 & 10 & 11 \\
Total & 54 & 24 & 78 \\
\hline
\end{tabular}

rapidly became more prominent (Fig. 1e). Each secondary inflorescence meristem functioned as the apical meristem of a secondary branch and started to produce tertiary inflorescence meristems, which in turn became the apical meristems of tertiary branches. Under the microscope, numerous arrested secondary inflorescence meristems were observed regularly arranged around the main inflorescences, while the inflorescence meristems remained terminal (Fig. 1i). In addition, distinct stratified layers were easily recognized in the inflorescence meristems. These observations indicate that the inflorescence meristems of smooth curds were arrested and did not produce floral meristems. The first- and higher-order inflorescence meristems of coarse curds looked similar to those of smooth curds (Fig. 1f). They differed in that the cauline leaves grew vertically and protruded from the curd surfaces, resulting in coarse curd surfaces where naked floral meristems were visible (Fig. 1j). In contrast, on the surfaces of granular curds, inflorescences of different orders developed further and produced floral meristems (Fig. 1g). Although sepal, petal, stamen, and pistil primordia were initiated, they failed to develop into floral organs, and this interesting phenomenon suggested that floral meristems were arrested. Under the microscope, numerous arrested floral meristems were observed around the main inflorescences, with some cauline leaves protruding between branch inflorescences and arising from the curd surface like thorns (Fig. 1k). Many floral meristems appeared on the surface of hairy curds (Fig. 1h). The floral organs were initiated and developed into tiny floral buds; however, these floral buds stopped growing and resembled hairs, indicating that the floral organs were arrested. Under the microscope, arrested floral organs and aberrant floral buds were clearly visible (Fig. 11).

The cauliflower development process can be broadly divided into seedling, curd formation, and flowering stages. In the field, curds that were fully mature before winter did not increase further in size. The following spring, a few inflorescence meristems began to develop and gave rise to new floral meristems on their periphery. The main and secondary inflorescence meristems in smooth curds elongated without the formation of cauline leaves (Fig. 1m, q), concurrent with the differentiation of a few floral buds. In coarse curds, the elongation of inflorescence meristems subtending cauline leaves and the differentiation of a few floral buds took place along with the growth of cauline leaves (Fig. 1n, r). Granular curds resumed development following floral meristem arrest and generated numerous floral buds that later flowered (Fig. 1o, s). When the floral meristems and internodes of inflorescences within curds elongated, the curds became "loose", and a few floral meristems developed into floral primordia, signaling the curd bolting stage. Floral meristems developed slowly but continuously, and sepals, petals, carpals, and stamens then began to form in preparation for normal flowering. At the same time, the curd surface became green because of the appearance of green sepals. Hairy curds resumed development following floral organ arrest and quickly flowered (Fig. 1p, t).

Plants of different accessions were transplanted into a growth chamber (16/8-h photoperiod with irradiance of $200 \mu \mathrm{mol}$ photons $/ \mathrm{m}^{2} / \mathrm{s}$ and a growth temperature of $25^{\circ} \mathrm{C}$ ) for further development. Under these conditions, which were conducive to flowering, the arrest of inflorescence meristems, floral meristems and floral organs was gradually broken. In $s c$ and $c c$ accessions, floral stems elongated, and a few floral buds developed, while most of the original inflorescence meristems remained suppressed or withered at the stage of primary protuberance (Fig. $1 \mathrm{u}$, v). In contrast, inflorescence elongation in $g c$ and $h c$ accessions was concurrent with the development of floral organs (Fig. 1w, x).

\section{Variation in BobCAL alleles}

To detect the mutations of BobCAL alleles in cauliflower accessions, we performed an extensive PCR analysis to amplify BobCAL genes in the genomes of $s c, c c, g c$, and $h c$ accessions. Some accessions were found to be heterozygous. To obtain homologous genotypes to achieve the highest discriminatory accuracy, all accessions were self-fertilized for more than five generations, and each accession was represented by one inbred line.

To examine the variations in $B o b C A L$ alleles, we cloned their coding regions using a primer pair (BobCAL_F/ BobCAL_R; Supplementary Table 1) designed to span the complete coding sequence. We identified 266 variant sites in the coding sequences of BobCAL alleles and 19 singlenucleotide polymorphisms (SNPs) across the collection of 78 accessions (Supplementary Table 2). The most interesting SNP was SNP451, which varied between $\mathrm{G}$ and T: while BobCAL containing 451G (referred to as Bob$C A L_{-} G$ ) encoded a full-length protein, that containing 
451T (BobCAL_T) encoded a truncated version because of a premature stop codon introduced by the SNP.

Among 57 accessions with tightly compact curds, 92\% (38/41) of $s c$ accessions and 94\% (15/16) of $c c$ accessions were found to carry $451 \mathrm{~T}$ (Table 1). In contrast, all (10) $g c$ accessions and 91\% (10/11) of $h c$ accessions harbored 451G. These results suggest that the 451T SNP is highly associated with tightly compact curds, while $451 \mathrm{G}$ is correlated with loosely compact curds. To our surprise, a few $s c$ and $c c$ accessions contained 451G, and one $h c$ accession possessed $451 \mathrm{~T}$, revealing that $451 \mathrm{G}$ is not the only genetic factor responsible for the loosely compact curd phenotype.

\section{Alternative splicing of BobCAL genes in cauliflower}

The BobCAL gene contains 8 exons encoding 251 amino acids. BobCAL alleles in different cauliflower accessions exhibited very similar structures and gene sequences. The BobCAL_G gene was found to encode a full-length protein, whereas BobCAL_T harbored a premature termination codon in exon 5 due to the $451 \mathrm{~T}$ SNP. To determine the relationship between the gene sequences and curd morphotypes of different accessions, we performed extensive cloning and sequencing of BobCAL cDNAs.

The coding sequences of BobCAL_G alleles in accessions with loosely compact curds were found to contain two AS sites (GenBank: AY514052-AY514055) (Fig. 2). One type of AS involved the retention of intron 4, which introduced a premature termination codon after amino acid 148. The other AS variant was an AS donor of intron 7 , located 6 bp downstream from the regular $3^{\prime}$ splice site of intron 7, which would introduce another premature termination mutation at position 219. The AS of Bob$C A L_{-} G$ was found to occur at introns 4 and 7 , either individually or in combination, to yield four splice variants: BobCAL_Ga,_Gb, Gc and _Gd. BobCAL_Ga (variant $a$ of $B o b C A L_{-} G$ ), comprising $756 \mathrm{bp}$, was predicted to encode a full-length protein of 251 amino acids, while the 762-bp BobCAL_Gb (variant $b$ ) encoded a 218-aminoacid putative protein; the 447-bp BobCAL_Gc (variant $c$ ) retained intron 4 and encoded a putative protein of 148 amino acids; and BobCAL_Gd (variant d), consisting of $447 \mathrm{bp}$, encoded a putative protein of 148 amino acids as well. Similarly, the four types of BobCAL_T AS variants perfectly corresponded to those of $B o b C A L_{-} G$, except that BobCAL_Tb encoded a putative protein consisting of 150 amino acids, which was 2 amino acids shorter than that encoded by BobCAL_Ta.

To examine the types of the AS of BobCAL alleles, we performed RT-PCR using strand-specific primers designed to amplify AS variants (Supplementary Table 1). We detected three or four types of AS variants in each accession, with most accessions exhibiting four (Table 2).
Given that BobCAL_Ga and BobCAL_Gb were functional while BobCAL_Gc and BobCAL_Gd were not, we wondered whether BobCAL_Ga and BobCAL_Gb were related to the granular and hairy curd morphotypes. All $20 \mathrm{gc}$ and $h c$ accessions possessed the BobCAL_Ga and BobCAL_Gb alleles; however, three or four types of AS variants were evident in $g c$ accessions, which was also found in $h c$ accessions (Supplementary Table 2). Different AS variants could thus be ruled out as the molecular factor responsible for the granular and hairy curd morphotypes.

\section{Expression levels of splice variants of BobCAL genes}

The $C A L$ gene is strongly expressed in the inflorescence meristems of Arabidopsis ${ }^{27}$. To further clarify the relationship between the AS of BobCAL and curd morphotypes, we collected RNA samples from the shoot tips of cauliflower plants at the curd induction stage and analyzed the expression levels of splice variants in the different accessions by RT-PCR. In $g c$ and $h c$ plants, RT-PCR amplification using primer pairs spanning the complete BobCAL_G coding sequence (Supplementary Table 3) yielded two bands (Fig. 3a). For the expression analysis of each splice variant, we used BobUBQ as the internal control and performed more than three repetitions of the RT-PCR experiments to ensure that the RT-PCR methods reproducibly determined the expression levels, and the expression levels could be experimentally validated. The sequencing of these PCR products revealed that the large band corresponded to BobCAL_Gc and BobCAL_Gd, while the small band consisted of BobCAL_Ga and Bob$C A L \_G b$. In $g c$ plants, the small band was weaker than the large band, indicating that the combined number of BobCAL_Ga and BobCAL_Gb transcripts $(a+b)$ was apparently less than that of BobCAL_Gc and BobCAL_Gd $(c+d)$. In $h c$ plants, however, the small band was stronger than the large band, indicating that the combined number of BobCAL_Ga and BobCAL_Gb transcripts $(a+b)$ was greater than that of BobCAL_Gc and BobCAL_Gd $(c+d)$. These results suggest that the expression levels of the BobCAL_Ga and BobCAL_Gb transcripts $(a+b)$ relative to the BobCAL_Gc and BobCAL_Gd $(c+d)$ transcripts in $h c$ accessions are much higher than in $g c$ accessions.

In all $h c$ accessions except for $h c-6$, the ratios of BobCAL_G $(a+b)$ to $(c+d)$ were $>1.5$ (Table 3 ), meaning that the $h c$ accessions were associated with relatively high expression of BobCAL_Ga and BobCAL_Gb transcripts $(a+b)$. hc-6 was exceptional in that the ratio of Bob$C A L_{-} T(a+b)$ to $(c+d)$ was $>1.5$. In the $g c$ accessions, the ratios of $(a+b)$ to $(c+d)$ were $\leqq 1.5$, indicating that $g c$ accessions were associated with relatively low expression of BobCAL_Ga and BobCAL_Gb transcripts $(a+b)$. In $s c$ and $c c$ plants, RT-PCR also produced two bands, where the smaller band was weaker than the larger one. The larger band was composed of BobCAL_Tc and 


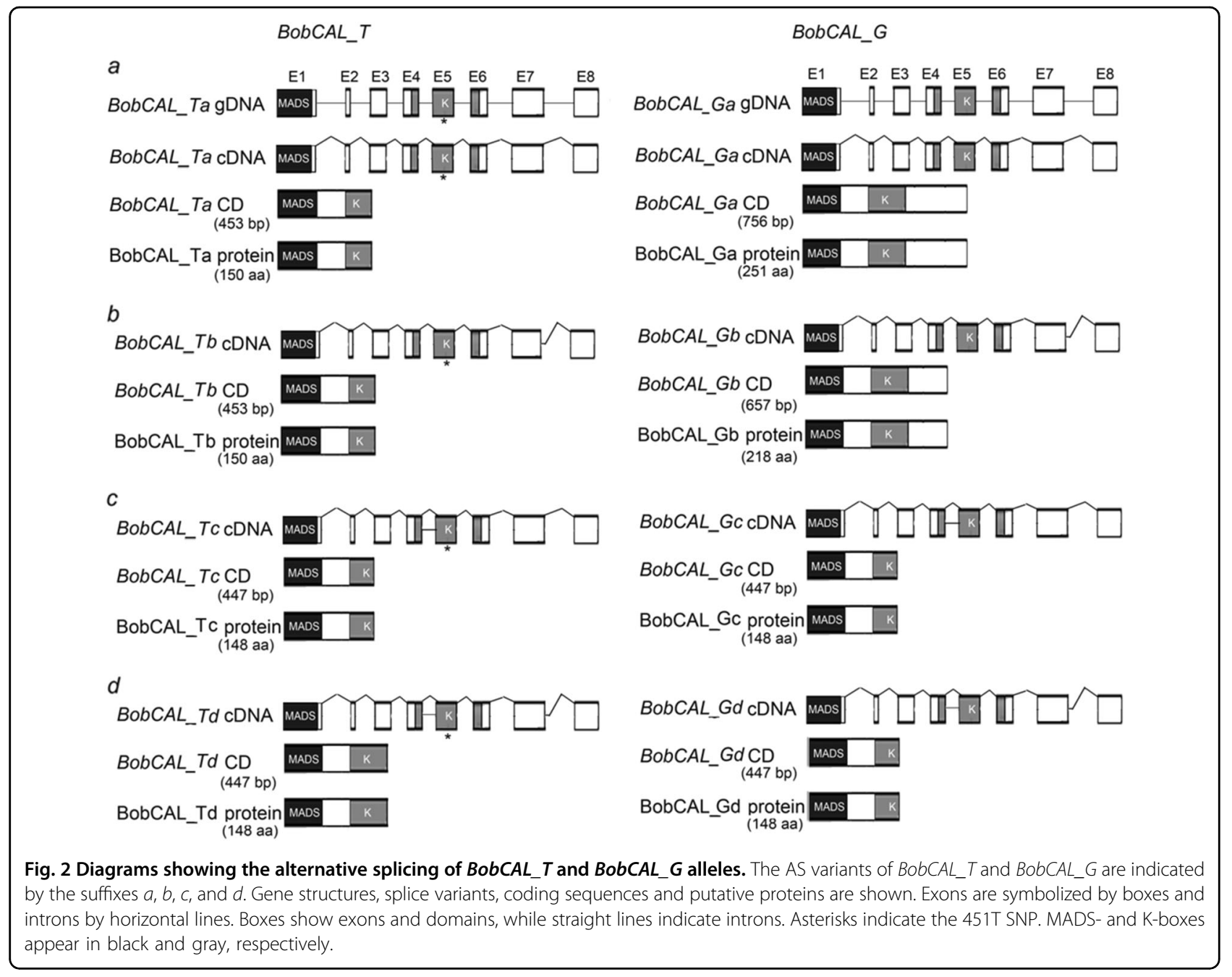

Table 2 Number of different alternative splice variants detected in $\mathbf{7 8}$ cauliflower accessions.

\begin{tabular}{lllllll} 
Curd phenotypes & $\boldsymbol{a}, \boldsymbol{b}, \boldsymbol{c}, \boldsymbol{d}$ & $\boldsymbol{a}, \boldsymbol{b}, \boldsymbol{d}$ & $\boldsymbol{a}, \boldsymbol{b}, \boldsymbol{c}$ & $\boldsymbol{b}, \boldsymbol{c}, \boldsymbol{d}$ & $\boldsymbol{a}, \boldsymbol{c}, \boldsymbol{d}$ & Total \\
\hline Smooth & 33 & 3 & 2 & 1 & 2 & 41 \\
Coarse & 14 & 1 & 0 & 0 & 1 & 16 \\
Granular & 8 & 0 & 1 & 1 & 0 & 10 \\
Hairy & 9 & 0 & 0 & 1 & 1 & 11 \\
Sum & 64 & 4 & 3 & 3 & 4 & 78 \\
\hline
\end{tabular}

BobCAL_Td, and the short band corresponded to Bob$C A L_{-} T a$ and BobCAL_Tb. The combined number of BobCAL_Tc and BobCAL_Td transcripts $(c+d)$ was apparently greater than that of BobCAL_Ta and Bob$C A L_{-} T b(a+b)$ transcripts. Among all $s c$ accessions except for $s c-3$ and $s c-14$, approximately half of the accessions presented ratios of BobCAL_T $(a+b)$ to $(c+$ d) $>1.5$, while the other half presented ratios $\leqq 1.5$
(Table 3); the same was true among all $c c$ accessions except for $c c-10$. Accessions $s c-3, s c-14$, and $c c-10$ harbored BobCAL_G alleles. These results indicated that neither $s c$ nor $c c$ accessions were associated with relatively high expression of BobCAL_Ta and BobCAL_Tb transcripts $(a+b)$.

Real-time PCR showed that BobCAL_Ga and Bob$C A L \_G b(a+b)$ expression levels in $h c$ plants were significantly higher than those of $g c$ plants (Fig. 3b), while $(c+d)$ expression levels were significantly lower than those of $g c$ plants. Importantly, higher expression levels of $(a+b)$ variants were significantly associated with the hairy curd morphotype (Fig. 3c), while lower expression levels were significantly related to granular curds. These results strongly suggest that the relative expression levels of $B o b C A L_{-} G a$ and $B o b C A L_{-} G b$ determine the granular and hairy curd morphotypes.

To investigate whether the total abundance of the different AS transcripts was the same in $g c$ and $h c$ plants, we performed RT-PCR using the pair of primers spanning 

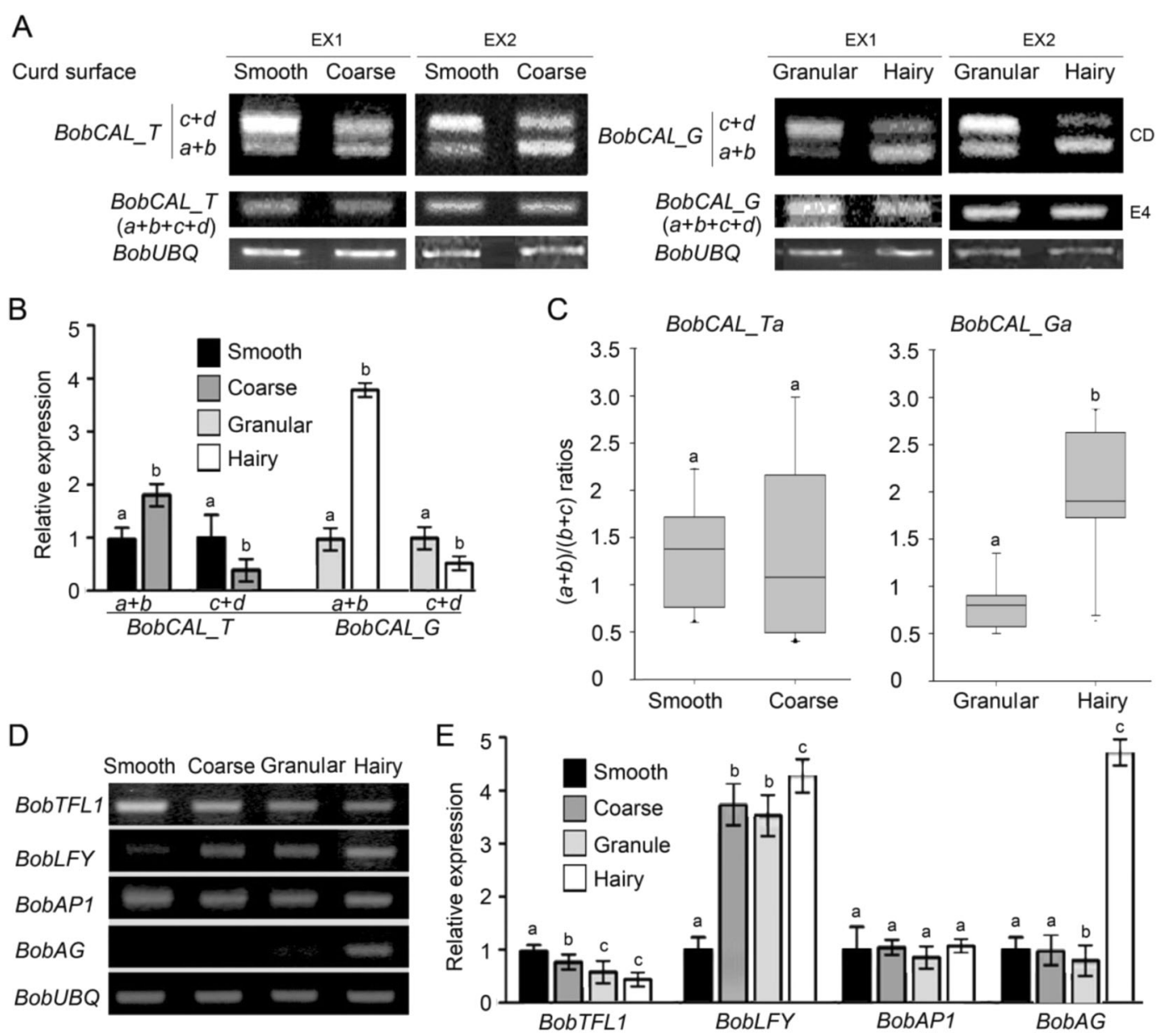

Fig. 3 Expression of BobCAL splice variants and BobCAL-related genes in cauliflower accessions. a Gels showing the four types of BobCAL splice variants in smooth (sc-2), coarse (cc-1), granular (gc-1) and hairy (hc-1) accessions. The $(a+b)$ band includes variants $a$ and $b$, while the $(c+d)$ band includes variants $c$ and $d$. EX1, experiment 1; EX2, experiment 2; CD, coding sequence; $E 4$, the fourth exon. $\mathbf{b}$ Expression levels of variant pairs $(a+b)$ and $(c+d)$. Three biological replicates of quantitative PCR were performed for each gene. The relative transcript level of each gene was normalized to that BobUBQ (BobUBIQUITIN) CDNA level for quantification. $\mathbf{c}$ Boxplot analysis showing the association between the $(a+b) /(c+d)$ ratios of BobCAL_T and BobCAL_G. All data are presented as the mean \pm SEM ( $n=10$ accessions). d Gels showing the expression levels of BobCALrelated genes in four types of cauliflower accessions. BobUBQ was used as an internal control. e Real-time PCR showing the relative expression of BobCAL-related genes in four types of cauliflower accessions. Three biological replicates of quantitative PCR were performed for each gene. The relative transcript level of each gene was normalized to the BobUBQ CDNA for quantification. Error bars indicate the standard error. Clusters with the same letters are not significantly differentiated from each other at the 0.01 level according to the $t$-test.

exon 4 of the BobCAL_G alleles (Supplementary Table 1). The expression levels obtained using these primers were expected to reflect total abundance of the transcripts of all four AS variants in the studied plants. In the $s c$ accessions, the total transcript levels of the four AS variants were roughly equal to those in the $c c$ accessions (Fig. 3a). Similarly, total transcript levels were generally the same between $g c$ and $c c$ accessions.

To answer the question of whether the abundance of $(a+b)$ relative to $(c+d)$ affects curd morphotypes, we analyzed the difference between the populations of different curd morphotypes through boxplot analysis. We randomly chose 10 accessions from each population to compare $(a+b) /(c+d)$ ratios. The difference in the $(a+$ $b) /(c+d)$ ratios of BobCAL_T between the $s c$ and $c c$ populations was not significant, indicating that smooth curds could not be discriminated from coarse curds according to the $(a+b) /(c+d)$ ratios of BobCAL_T (Fig. 3c). In contrast, the difference in the $(a+b) /(c+d)$ ratios of BobCAL_G between the $g c$ and $h c$ populations was significant, indicating that hairy curds could be discriminated from granular curds according to the $(a+b) /$ $(c+d)$ ratios of BobCAL_G. This result implies that BobCAL_Ta and BobCAL_Tb have lost their original function, probably because of the G451T mutation, whereas BobCAL_ga and BobCAL_Gb are functional, and 
Table 3 Association between curd phenotypes and the ratio of the expression levels BobCAL variants $(a+b)$ versus $(c+d)$.

\begin{tabular}{|c|c|c|c|c|c|c|}
\hline \multicolumn{2}{|c|}{ Splicing variants } & \multicolumn{4}{|c|}{ Number of accessions } & \multirow[t]{2}{*}{ Sum } \\
\hline Alleles & $(a+b) /(c+d)$ & Smooth & Coarse & Granular & Hairy & \\
\hline \multirow[t]{2}{*}{ BobCAL_T } & $>1.5$ & 18 & 8 & 0 & 1 & 27 \\
\hline & $\leqq 1.5$ & 20 & 7 & 0 & 0 & 27 \\
\hline \multirow[t]{2}{*}{ BobCAL_G } & $>1.5$ & 2 & 1 & 0 & 11 & 14 \\
\hline & $\leqq 1.5$ & 0 & 0 & 10 & 0 & 10 \\
\hline Total & & 40 & 16 & 10 & 12 & 78 \\
\hline
\end{tabular}

the amount of BobCAL_G $(a+b)$ relative to BobCAL_G $(c+d)$ is responsible for the granular or hairy morphotypes.

\section{Expression levels of other floral identity genes}

The alterative splicing of BobCAL may alter the expression patterns of related meristem identity genes. To evaluate the relationship between BobCAL and major meristem identity genes, we studied the expression patterns of the meristem genes BobTFL1, BobLFY and $B o b A P 1$ and the floral organ identity gene BobAG.

At the curd induction stage, BobTFL1 was strongly expressed in $s c$ accessions but was weakly detected in $h c$ plants (Fig. 3d, e). In contrast, BobLFY was weakly expressed in $s c$ accessions and strongly expressed in $h c$ accessions. In Arabidopsis, the inhibition of TFL1 requires $\mathrm{CAL} / \mathrm{AP}^{27}{ }^{27}$. The strong expression of BobTFL1 in $s c$ accessions means that the truncated proteins encoded by BobCAL_T cannot function to inhibit BobTFL1. Conversely, the weak expression of BobTFL1 in $h c$ accessions indicates that BobCAL_Ga does inhibit BobTFL expression to some extent. In the $s c$ accessions, BobTFL1 was strongly expressed at the curd initiation stage, which indicates that early inflorescence meristems are the sites of BobTFL1 expression in the absence of BobCAL activity. The expression levels of BobLFY in the $g c$ and $h c$ accessions were much higher than those in the $s c$ accessions. BobAP1 expression was almost the same in the four groups of accessions.

\section{Gain of function of BobCAL_G in smooth cauliflower}

The premature termination codon (GAG $\rightarrow$ TAG) in the fifth exon of the BobCAL gene has been deduced to affect the curd morphotype of cauliflower ${ }^{9,10}$, but direct experimental evidence of this effect has been lacking. To support this deduction, a functional BobCAL_Ga variant from $h c-1$, a representative inbred line of the $h c$ accessions, was constructed from the T-DNA of a binary vector under the control of the CaMV 35S promotor and then introduced into $s c$-1, a representative $s c$ inbred line. While the inflorescences of $s c-1$ plants were severely arrested and subsequently formed smooth curds, the inflorescences of the transgenic $\mathrm{Bc}-1$ and $\mathrm{Bc}-2$ lines were not fully arrested and failed to yield smooth curds (Fig. 4a-c). These plants instead produced floral meristems immediately after floral initiation and underwent floral bud differentiation, which resulted in an $h c$-like curd phenotype. In both transgenic lines, green inflorescences were generated from the shoot apices of the plants, and inflorescence and floral meristem arrest was consequently not obvious. In terms of appearance, the inflorescences of the $\mathrm{Bc}-1$ line exhibited a greater number of developed green floral buds than did those of the Bc-2 line; they were visually similar to cabbage inflorescences but more branched and dwarfed. Under a scanning electron microscope, we observed that the flower buds of transgenic cauliflower exhibited developing floral organs on their shoot apices, whereas the inflorescence meristems of control plants remained arrested. The most striking difference was that $\mathrm{Bc}-1$ and $\mathrm{Bc}-2$ plants did not require low temperatures for vernalization and began flowering in autumn, while $s c-2$ plants underwent vernalization during winter and began flowering the following spring. These results indicate that the smooth curd morphotype caused by $B o b C A L_{-} T$ is partially complemented by BobCAL_Ga.

The transformed plants were self-fertilized for two generations, and two transgenic lines for the BobCAL_Ga gene were obtained. Southern hybridization confirmed that a single copy of the transgene had been inserted into the cauliflower genome (Fig. 4d). The expression of the $B o b C A L$ gene in cauliflower was analyzed by examining the transcripts of BobCAL_Ga in Bc-1 and Bc-2 plants in shoot apices at the curd induction stage by RT-PCR. Two bands, corresponding to 790- and 920-bp fragments, were resolved in an agarose gel (Fig. 4e). The shorter fragments, whose lengths were consistent with BobCAL_Ga cDNAs, exhibited higher expression than $s c-1$, whereas the longer fragments, corresponding to BobCAL_Tc and Bob$C A L_{-} T d$ cDNAs, displayed lower expression levels. These results suggest that exogenous BobCAL_Ga was overexpressed in $s c-1$ plants (Fig. $4 \mathrm{f}$ ). At the same time, the reduced expression of BobCAL_Tc and BobCAL_Td in $\mathrm{Bc}-1$ and $\mathrm{Bc}-2$ plants implied that the overexpression of BobCAL_Ga decreased the expression levels of Bob$C A L_{-} T c$ and BobCAL_Td. We conclude that BobCAL_Ga conferred a floral development function in smooth curd accessions.

$B o b L F Y$ and BobAG were upregulated in $\mathrm{Bc}-1$ and $\mathrm{Bc}-2$ plants (Fig. 4f, g), revealing that BobCAL_Ga positively regulates BobLFY and BobAG in cauliflower. The transition from a smooth curd morphotype to a hairy-like morphotype may be caused by the upregulation of $B o b L F Y$ and BobAG. 


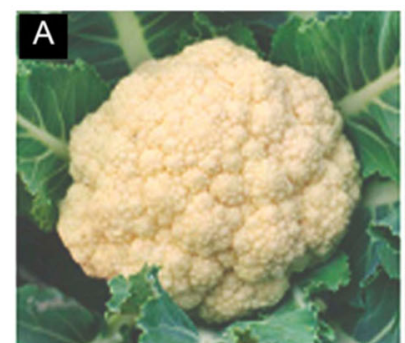

D
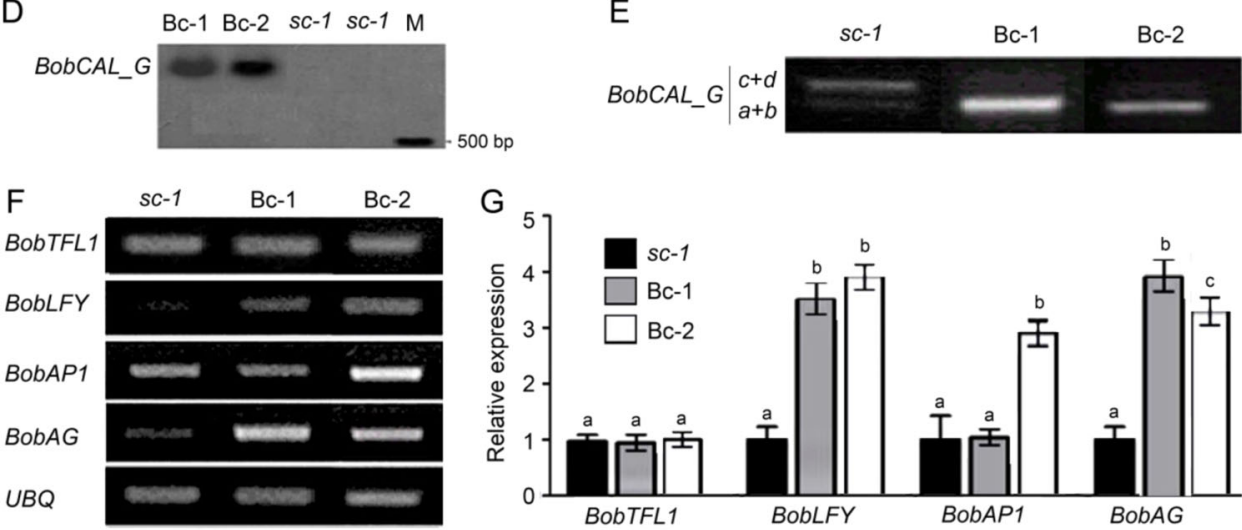

Fig. 4 Expression of $p 355:: B o b C A L \_G a$ in transgenic cauliflower and expression of BobCAL splice variants in wild-type plants a Wild-type plant with smooth curds. Transgenic Bc-1 plant (b) with hairy-like curds. c Transgenic Bc-2 plant with hairy-like curds. d Southern hybridization results for transgenic plants using a CaMV 355 promoter fragment labeled with 32P-dCTP as a probe. Lane M is a molecular size marker. Comparisons of the expression levels of BobCAL splice variants $(\mathbf{e})$ and BobCAL-related genes $(\mathbf{f}, \mathbf{g})$ in transgenic plants based on real-time PCR. The relative transcript level of each gene was normalized to the BobUBQ CDNA level for quantification. Error bars represent the SE (standard error) calculated from three biological replicates, each of which included three technical replicates. $a$ and $b$ indicate significant differences at the 0.05 and 0.01 levels, respectively, according to the $t$-test.

\section{Discussion}

Granular and hairy curd morphotypes are relevant to the AS of BobCAL-G

In plants of $A$. thaliana, floral meristems are produced from inflorescence meristems after floral induction ${ }^{28,29}$. In cauliflower, inflorescence meristems are temporarily arrested, as they fail to produce floral meristems, with numerous inflorescence meristems then appearing on the curd surface. We found that these events occur in tightly compact curds whose inflorescence meristems are naked and arrested. Unlike smooth and coarse curds, granular curds include numerous floral meristems that are temporarily arrested, while hairy curds retain the state of floral buds on the curd surface. Most likely, floral meristem arrest occurs in granular curds after the cessation of inflorescence meristem arrest, whereas hairy curds experience floral organ arrest after the interruption of inflorescence and floral meristem arrest. The inflorescence meristem arrest of granular and hairy curds is thus of shorter duration than that of smooth and coarse curds.

The mutation of BobCAL was previously considered to be strongly associated with the curd phenotype ${ }^{24}$. However, the goodness-of-fit test rejected this model by showing that BobCAL alleles were poor predictors of curd phenotypes $^{26}$. The contradiction about the role of Bob$C A L$ in curd formation apparently arose because the surveyed accessions with different curd morphotypes were mixed together. We discriminated accessions with hairy and granular curds from those with smooth and coarse curds and found that smooth and coarse curd morphotypes are highly associated with the 451T SNP (BobCAL_T), whereas hairy and granular curd morphotypes are correlated with 451G (BobCAL_G). Although $s c$ and $c c$ accessions differ in regard to the presence of cauline leaves subtended in curds, they share the same G451T SNP. In theory, BobCAL_T alleles encode putative truncated proteins in $s c$ and $c c$ accessions, whose inflorescence meristems are arrested irrespective of the splice variants of BobCAL_T. In contrast, BobCAL_G alleles are expected to encode normal proteins in $h c$ and $g c$ accessions, whose floral meristems and floral organs are arrested in a manner dependent on the splice variants of BobCAL_G. Thus, the AS of BobCAL_G is crucial for the granular and hairy curd morphotypes of $g c$ and $h c$ accessions. However, the hairy phenotype of $h c-6$ is not linked to BobCAL_G. In $h c-6$ plants, the ratio of 
BobCAL_T $(a+b)$ to $(c+d)$ is $>1.5$. Most likely, the altered function of the other genes can compensate for the loss of BobCAL function in this accession. On the other hand, the smooth and coarse phenotypes of accessions $s c-3, s c-14$, and $c c-10$ are not linked to BobCAL_T. We suggest that the splice variants of BobCAL_G are not the sole reason for the occurrence of hairy curds and that the S451T SNP is not the sole element responsible for the occurrence of smooth and coarse curds.

We observed curd surfaces at the time of curd maturity that is ideal for harvesting. At that time, the accessions with compact and smooth curds had not shown any riceyness. However, some compact and smooth curds tended toward certain degrees of riceyness post harvest in spring. "Riceyness" refers to precocious flower bud initiation on the curd surface of cauliflower, which is undesirable for the market ${ }^{30}$. The difference in the degree of riceyness in compact and smooth curds is reflected by the distribution frequency of the flowering time. The plants within the whole population flowered earlier on average in 2016 than in 2017. This implies that riceyness and curd compactness are affected by the interaction between genes and environmental elements, as flowering times are altered under different growing conditions.

Relative levels of BobCAL_Ga and BobCAL_Gb transcripts define the fates of floral meristem and floral organ arrest

Among the accessions with BobCAL_G alleles in our study, the total expression levels of the four AS variants in plants with granular curds were almost equal to those in hairy curd plants. This observation strongly suggests that BobCAL_G genes in $g c$ and $h c$ accessions show only partial activities. If the BobCAL_G genes were fully functional, the inflorescences would not have been arrested and would have developed into normal flowers, as observed in cabbage. If the BobCAL_G genes had completely lost their functionality, the inflorescences would have been arrested, as observed in smooth curds. Compared with $s c$ and $c c$ accessions, which are characterized by inflorescence meristem arrest, $g c$ and $h c$ accessions are marked by floral meristem arrest and floral organ arrest, respectively, apparently because of the partial function of BobCAL_G alleles.

The total expression levels of the four different $B o b$ $C A L_{-} G$ AS transcripts are roughly equal between $h c$ and $g c$ plants. In this situation, any change in the expression level of one AS variant should affect the expression levels of the others. In $g c$ accessions, both BobCAL_Ga and $B o b C A L_{-} G b$ are expressed at lower levels than Bob$C A L_{-} G c$ and $B o b C A L_{-} G d$, and their levels are sufficient to overcome inflorescence meristem arrest but not floral meristem arrest. In $h c$ accessions, BobCAL_Ga and Bob$C A L_{-} G b$ are expressed at levels higher than $B o b C A L_{-} G c$ and $B o b C A L_{-} G d$, and their expression is sufficient to overcome both inflorescence and floral meristem arrest but not floral organ arrest. The relative level of BobCAL_G $(a+b)$ determines the fate of inflorescence meristem arrest, floral meristem arrest and floral organ arrest and, thus, the curd morphotype. Granular and hairy curd morphotypes are caused by floral meristem arrest and floral organ arrest, respectively.

Under normal conditions, the expression levels of BobCAL_G $(a+b)$ relative to BobCAL_G $(c+d)$ are stable in each $h c$ and $g c$ accession. When BobCAL_Ga and $B o b C A L \_G b$ transcripts are predominantly expressed, floral organ arrest occurs rather than floral meristem arrest. When the expression of BobCAL_Gc and Bob$C A L_{-} G d$ transcripts is predominant, the opposite is true. We verified this outcome via the genetic transformation of BobCAL_Ga in cauliflower. The overexpression of BobCAL_Ga decreased the expression levels of Bob$C A L_{-} T c$ and BobCAL_Td and caused a transition from inflorescence meristem arrest to floral organ arrest. In the field, environmental conditions such as temperature and photoperiod usually affect curd morphotypes and cauliflower yield in $g c$ and $h c$-like cultivars, probably by influencing the expression levels of BobCAL_G $(a+b)$ relative to BobCAL_G $(c+d)$.

The question arises of how $g c$ and $h c$ accessions control the expression levels of BobCAL_Ga and BobCAL_Gb relative to those of $B o b C A L_{-} G c$ and $B o b C A L_{-} G d$. One possibility is that some elements upstream of $B o b C A L_{-} G a$ and $B o b C A L_{-} G b$ variants differentially regulate one or more AS events. Alternatively, the partial or complete retention of introns 4 and/or 7 in BobCAL_Gc and BobCAL_Gd may influence their expression in some accessions.

\section{Curd morphotypes of cauliflower are regulated by the AS of BobCAL alleles}

In Arabidopsis, an increase in $L F Y$ expression and the consequent suppression of TFL1 initiates flowering via the upregulation of AP1 and CAL. ap1 cal double mutants have a 'cauliflower' appearance ${ }^{11}$, while the corresponding single mutants do not. If cauliflower plants form curds in the same manner, the activity of $A P 1$ must not be complete, as a single $B o b C A L$ mutation is insufficient to alter the curd phenotype. Therefore, it is important to clarify whether BobAP1 is fully activated in different cauliflower accessions.

In cauliflower plants, inflorescence meristem arrest is the primary cause of smooth and coarse curd morphotypes, while floral meristem arrest and floral organ arrest are responsible for granular and hairy curd morphotypes, respectively. BobCAL_T is nearly fixed in $s c$ and $c c$ accessions, and the same is true for BobCAL_G in $g c$ and $h c$ accessions. Either BobCAL_T or BobCAL_G alleles generate the four types of splice variants: $a, b, c$, and $d$. The predominant expression of BobCAL_Ga and/or BobCAL_Gb 
favors the hairy curd morphotype, whereas the predominant expression of BobCAL_Gc and/or BobCAL_Gd results in granular curds. To clarify the genetic relationship between curd morphotypes and the relative expression levels of BobCAL variants, their $(a+b) /(c+d)$ ratios were compared. We found that the accessions with BobCAL_T showed smooth and/or coarse curds regardless of the ratio of $(a+b) /(c+d)$, whereas the accessions with BobCAL_G exhibited granular or hairy curds. When the $(a+b) /(c+d)$ ratio of BobCAL_G is $>1.5$, all accessions show hairy curds, and when the ratio is $\leqq 1.5$, all accessions show granular curds. Clearly, the $(a+b) /(c+d)$ ratio of BobCAL_G is relevant to the fate of hairy and granular phenotypes.

On the basis of our genetic and expression analysis results, we propose a model for the genetic regulation of $B o b C A L$ gene AS to explain the curd phenotypes of cauliflower (Fig. 5). In this model, the BobCAL genes present two copies of BobCAL_T and BobCAL-G, each of which shows four types of AS variants. BobCAL_T mainly exists in $s c$ and $c c$ accessions, while BobCAL-G is found in $g c$ and $h c$ accessions. Smooth and/or coarse curds are associated with BobCAL_T regardless of the ratio of $(a+b) /(c+d)$, whereas granular and hairy curds are associated with $B o b$ $C A L_{-} G$. In the latter case, higher ratios of $(a+b) /(c+d)$ are associated with hairy curds, while lower ratios are associated with granular curds. The $(a+b) /(c+d)$ ratios of BobCAL_G determine the fate of hairy and granular curds.

The plants with the four types of curds show differences in vernalization to initiate flowering. The $s c-2$ plants with smooth curds flower latest owing to the delayed transition from inflorescence-to-floral meristems. They require a longer period of vernalization for flowering. The transgenic $\mathrm{BC}-1$ and $\mathrm{Bc}-2$ plants do not require vernalization and bolt and flower before winter. This difference indicates that the relative abundance of BobCAL_Ga variants not only alters curd morphotypes in cauliflower but also affects the timing of the transition from inflorescence-tofloral meristems and therefore affects the fate of vernalization in cauliflower plants.

\section{Materials and methods}

\section{Plant materials and growth conditions}

A total of 78 cauliflower accessions with different curd phenotypes were used in this study. All accessions were self-fertilized for more than five generations to obtain inbred lines. Cauliflower seeds were sown in July, and the resultant seedlings were transplanted into the field one month after germination according to conventional practices at the SIPP Experimental Station, Shanghai, China. The field experiments were conducted in a randomized complete block design with two replications ${ }^{31}$ in replicated field trials for three successive growing seasons (July 2016-2018). More than 20 plants per accession were transferred into greenhouses in the subsequent January

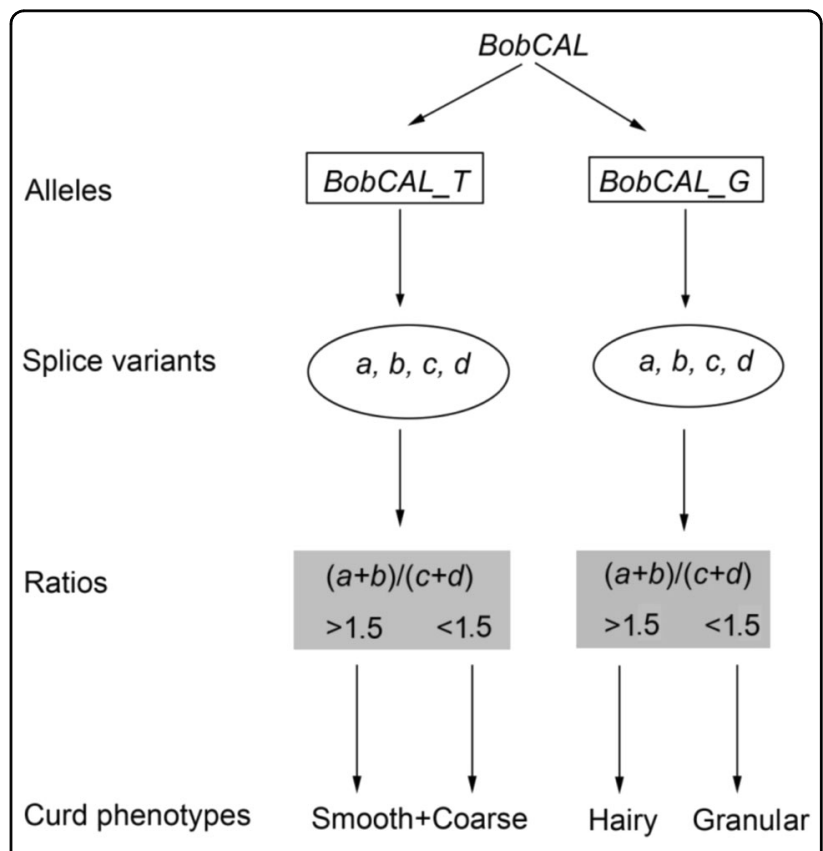

Fig. 5 Proposed model of the genetic regulation of cauliflower curd phenotypes by the alternative splicing of the BobCAL gene.

for further growth. All accessions were phenotyped for curd-related traits.

Transgenic cauliflower plants were grown simultaneously in the field in July 2017 and transplanted into a greenhouse in the following January. Two lines of $T_{1}$ generation transgenic cauliflower plants and their positive progenies were self-pollinated, and seeds were harvested at maturity. For segregation testing, seedlings from the $T_{2}$ and $T_{3}$ generations were selected on agar-solid Murashige-Skoog (MS) medium with $50 \mathrm{mg} / \mathrm{L}$ kanamycin $(\mathrm{Km})$.

\section{Phenotype characterization}

The stage of developmental arrest was scored on a fourpoint scale with a photographic reference card ${ }^{32}$ and coded into four phenotypes ranging from inflorescence meristem arrest (smooth and coarse curds) to floral meristem arrest (granular curds) and floral organ arrest (hairy curds). The number of days to flowering was counted from transplantation to the opening of the first flower bud in plants. The number of days to curd maturity was counted from transplantation to the harvest maturity of curds. The width of curds was measured to represent curd size.

\section{Scanning electron microscopy}

Shoot apices with developing leaves (5 $\mathrm{mm}$ in length) were harvested from the plants of different types at the curd induction stage. For scanning electron microscopy, the tissues were fixed in FAA ( $2 \%$ formaldehyde, $44.5 \%$ ethanol, and $6 \%$ acetic acid), dehydrated, critical point-dried with $\mathrm{CO}_{2}$, and sputter-coated with gold palladium. Specimens 
from at least three plants for each variant were observed and photographed with a JEOL JSM-6360LV scanning electron microscope.

\section{Vector construction}

A 0.8-kb EcoRI fragment containing BobCAL_Ga cDNA was cloned from an RNA sample isolated from the hairy morphotype accession $h c-1$ and subcloned into the EcoRI site of a pBluescript KS plasmid. The insertion orientation of BobCAL_Ga was verified by cleaving a pre-existing XhoI site in the gene. The BobCAL_Ga cDNA was then excised using BamHI and $\mathrm{KpnI}$ and inserted into a pJR1 binary vector between the CaMV $35 \mathrm{~S}$ promoter and the NOS terminator. The resulting plant expression vector, $p J R$ BobCAL_Ga, was subsequently introduced into Agrobacterium LBA4404 via the freeze-thaw method ${ }^{33}$.

\section{Generation of transgenic cauliflower}

Seeds of the smooth-morphotype inbred line $s c-1$ were surface sterilized and then germinated on agar-solid MS medium. Hypocotyl segments of 8-10-day-old seedlings were infected with LBA4404 containing $p J R-B o b C A L \_G a$ and cocultured for 3 days. The infected segments were then induced to produce $\mathrm{Km}$-resistant shoots on MS medium containing $3 \mathrm{mg} / \mathrm{L} 6-\mathrm{BA}, 0.5 \mathrm{mg} / \mathrm{L}$ IAA, $500 \mathrm{mg} /$ $\mathrm{L}$ carbenicillin and $50 \mathrm{mg} / \mathrm{L} \mathrm{Km}$. The positive shoots were induced to root on MS medium containing $0.5 \mathrm{mg} / \mathrm{L}$ IAA and then transplanted into the greenhouse.

Three $\mathrm{Km}$-resistant lines were identified as transgenic by PCR and Southern hybridization. The primers used for PCR amplification were npt-p1 (5'-GAGGCTATTCGG CTATGACTG-3') and npt-p2 (5'-ATCGGCAGCGGC GATACCGTA-3'), which were specific for the NPT-II gene conferring $\mathrm{Km}$ resistance in the vector pJR1. For Southern hybridization, $10 \mu \mathrm{g}$ of genomic DNA extracted from untransformed and $\mathrm{Km}$-resistant cauliflower leaves was digested with EcoRI and BamHI and then subjected to $1.0 \%$ agarose gel electrophoresis. A PCR-amplified fragment of the $35 \mathrm{~S}$ promoter labeled with ${ }^{32} \mathrm{P}$ was applied as a probe to detect plant genomic DNA following the procedure of Sambrook et al. .

\section{RNA and DNA extraction}

For the cDNA cloning and RNA isoform analysis of the BobCAL, BobLFY, BobAP1, and BobAG alleles, shoot tips with developing leaves were freshly harvested at the curd induction stage, and RNA samples were isolated from shoot tip tissues. For RT-PCR and real-time PCR, three plants of each accession at the same stage as mentioned above were randomly selected for the sampling of shoot tips with developing leaves, from which RNA samples were isolated. Ten accessions were randomly selected to perform real-time PCR for the discrimination of expression levels between the four groups of accessions.
Total RNA was extracted from shoot tips at the curd induction stage via the guanidinium isothiocyanate method $^{34}$. Total genomic DNA was extracted from cauliflower using a modified CTAB method ${ }^{35}$.

\section{RT-PCR}

Two micrograms of total RNA was reverse transcribed and amplified using an mRNA Selective Reverse Transcription 2.1 PCR kit (Takara, Dalian, China) according to the manual provided by the manufacturer ${ }^{34}$.

The primers used for the RT-PCR analysis of $U B Q$, BobTFL1, BobLFY, BobAP1, BobCAL, and BobAG are listed in Supplementary Table 1. The annealing temperatures used for PCR amplification were as follows: $54^{\circ} \mathrm{C}$ for BobTFL1, BobLFY, and BobAP1 and $56^{\circ} \mathrm{C}$ for BobCAL, BobAG, and $U B Q$. Specific primer pairs (Supplementary Table 3 ) were designed to perform real-time PCR in a MyiQ2 Two-color Real-time PCR Detection System (Bio-Rad, Richmond, CA, USA). For the expression analysis of each splice variant, the RT-PCR/real-time PCR experiments were performed more than three times, and the methods reproducibly determined the expression levels and experimentally validated the expression levels. At least three biological replicates of the quantitative PCR assays were performed for each gene. The relative transcript level of each gene was normalized to that of $B o b U B Q$ cDNA for quantification. $t$-tests were performed to analyze the significance of differences.

\section{Statistics analysis}

For real-time PCR, three biological replicates of the quantitative PCR assays were performed for each accession. The relative transcript level of each gene was normalized to the BobUBQ cDNA level for quantification ${ }^{34}$. Error bars indicate the standard error. For boxplot analysis, all data are presented as the mean $\pm \operatorname{SE}(n \geqq 10$ accessions). Clusters with the same letters are not significantly differentiated from each other at the 0.01 level according to the $t$-test ${ }^{36}$.

\section{Identification of BobCAL gene AS variants}

After the reverse transcription of $2 \mu \mathrm{g}$ of RNA using oligo-dT primers with reverse transcriptase (Takara), $5 \mu \mathrm{L}$ of the resulting products were subjected to PCR amplification with the BobCAL-F/BobCAL-R primers as described above. The electrophoresis of the products in a $1 \%$ agarose gel yielded two specific bands. Both bands were recovered and cloned into a pMD18-T vector (Takara) for sequencing.

\section{Sequence analysis}

All cDNA sequences (GenBank: AY514052-AY514055) were analyzed with DNAStar Lasergene sequence analysis software and then aligned in CLUSTAL X $1.8^{36}$. 


\section{Association analysis}

Box plots were drawn according to the method of Tukey $^{37}$. The median and quartile values of gene expression data of the cauliflower accessions were calculated using $\mathrm{R}$ statistical software. Significant differences between subpopulations were detected by the Kruskal-Wallis test ${ }^{38}$.

\section{Acknowledgements}

This work was supported by the National Programs for Science and Technology Development of China (Grant Nos. 2016YFD0101900 and 2016YFD100500), the Natural Science Foundation of China (Grant No. 31571261), and the Shanghai Agriculture Applied Technology Development Program, China (Grant Nos. G2015060107 and Z20160109).

\section{Author details}

${ }^{1}$ National Laboratory of Plant Molecular Genetics, Center for Excellence in Molecular Plant Sciences, Shanghai Institutes of Plant Physiology \& Ecology, Chinese Academy of Science, 300 Fenglin Road, Shanghai 200032, China. ${ }^{2}$ State Key Laboratory of Crop Stress Biology in Arid Areas and College of Agronomy, Northwest A\&F University, Yangling, Shaanxi, China. ${ }^{3}$ State Key Laboratory of North China Crop Improvement and Regulation, Department of Horticulture, Hebei Agricultural University, Baoding 071001, China

\section{Author contributions}

Y.H. conceived the project and research plan; W.C. and B.C. performed the experiments; X.W. performed the statistical analysis; Y.Z.X., S.H., and X.L. analyzed the data and wrote some parts of the article.

\section{Conflict of interest}

The authors declare that they have no conflict of interest.

Supplementary Information accompanies this paper at (https://doi.org/ 10.1038/s41438-020-00378-x)

Received: 7 January 2020 Revised: 19 July 2020 Accepted: 19 July 2020 Published online: 01 October 2020

\section{References}

1. Smyth, D. R. Flower development: origin of the cauliflower. Curr. Biol. $\mathbf{5}$ 361-363 (1995)

2. Kieffer, M. Fuller, M. \& Jellings, A. Mathematical model of cauliflower curd architecture based on biometrical analysis. Acta Hortic. 407, 361-368 (1996).

3. Sadik, S. Morphology of the curd of cauliflower. Am. J. Bot. 49, 290-297 (1962).

4. De Candolle, A. Prodromus (Treuttel and Wurtz, 1824).

5. Masters, M. T. On the structure of the flower in the genus Napoleona, \&c Botanical J. Linn. Soc. 10, 492-504 (1869).

6. Sadik, S. \& Ozbun, J. The association of carbohydrate changes in the shoot tip of cauliflower with flowering. Plant Physiol. 43, 1696-1698 (1968).

7. Anthony, R. G., James, P. E. \& Jordan, B. R. Cauliflower (Brassica oleracea var botrytis L.) curd development: the expression of meristem identity genes. J. Exp. Bot. 47, 181-188 (1996).

8. Carr, S. M. \& Irish, V. F. Floral homeotic gene expression defines developmental arrest stages in Brassica oleracea L. vars. botrytis anditalica. Planta 201, 179-188 (1997).

9. Bowman, J. L. et al. Control of flower development in Arabidopsis thaliana by APETALA1 and interacting genes. Development 119, 721-743 (1993).

10. Kempin, S. A., Savidge, B. \& Yanofsky, M. F. Molecular basis of the cauliflower phenotype in Arabidopsis. Science 267, 522-525 (1995).

11. Ferrandiz, C., Gu, Q., Martienssen, R. \& Yanofsky, M. F. Redundant regulation of meristem identity and plant architecture by FRUITFULL, APETALA1 and CAULIFLOWER. Development 127, 725-734 (2000).

12. Alvarez-Buylla, E. R., Garcia-Ponce, B. \& Garay-Arroyo, A. Unique and redundant functional domains of APETALA1 and CAULIFLOWER, two recently duplicated Arabidopsis thaliana floral MADS-box genes. J. Exp. Bot. 57, 3099-3107 (2006).

13. Pelaz, $\mathrm{S}$. et al. $\mathrm{B}$ and $\mathrm{C}$ floral organ identity functions require SEPALLATA MADS-box genes. Nature 405, 200-203 (2000).

14. Pelaz, $S$. et al. APETALA1 and SEPALLATA3 interact to promote flower development. Plant J. Cell Mol. Biol. 26, 385-394 (2001).

15. Samach, A. et al. Distinct roles of CONSTANS target genes in reproductive development of Arabidopsis. Science 288, 1613-1616 (2000).

16. Hartmann, U. et al. Molecular cloning of SVP: a negative regulator of the floral transition in Arabidopsis. Plant J. Cell Mol. Biol. 21, 351-360 (2000).

17. Krizek, B. A. \& Meyerowitz, E. M. Mapping the protein regions responsible for the functional specificities of the Arabidopsis MADS domain organ-identity proteins. Proc. Natl Acad. Sci. USA 93, 4063-4070 (1996).

18. Riechmann, J. L., Krizek, B. A. \& Meyerowitz, E. M. Dimerization specificity of Arabidopsis MADS domain homeotic proteins APETALA1, APETALA3, PISTILLATA, and AGAMOUS. Proc. Natl Acad. Sci. USA 93, 4793-4798 (1996).

19. Fan, H. Y., Hu, Y., Tudor, M. \& Ma, H. Specific interactions between the $K$ domains of $A G$ and $A G L S$, members of the MADS domain family of DNA binding proteins. Plant J. 12, 999-1010 (1997)

20. Mizukami, Y. et al. Functional domains of the floral regulator AGAMOUS: characterization of the DNA binding domain and analysis of dominant negative mutations. Plant Cell 8, 831-845 (1996).

21. Moon, Y.-H. et al. Determination of the motif responsible for interaction between the rice APETALA1/AGAMOUS-LIKE9 family proteins using a yeast two-hybrid system. Plant Physiol. 120, 1193-1204 (1999).

22. Yang, Y. \& Jack, T. Defining subdomains of the $K$ domain important for protein-protein interactions of plant MADS proteins. Plant Mol. Biol. 55, 45 (2004).

23. Huang, $\mathrm{H}$. et al. The Arabidopsis MADS-box gene AGL3 is widely expressed and encodes a sequence-specific DNA-binding protein. Plant Mol. Biol. 28 549-567 (1995).

24. Smith, L. B. \& King, G. J. The distribution of BoCAL-a alleles in Brassica oleracea is consistent with a genetic model for curd development and domestication of the cauliflower. Mol. Breed. 6, 603-613 (2000).

25. Purugganan, M. D., Boyles, A. L. \& Suddith, J. I. Variation and selection at the CAULIFLOWER floral homeotic gene accompanying the evolution of domesticated Brassica oleracea. Genetics 155, 855-862 (2000).

26. Labate, J. A., Robertson, L. D., Baldo, A. M. \& Björkman, T. Inflorescence identity gene alleles are poor predictors of inflorescence type in broccoli and cauliflower. J. Am. Soc. Hortic. Sci. 131, 667-673 (2006)

27. Ratcliffe, O. J., Bradley, D. J. \& Coen, E. S. Separation of shoot and floral identity in Arabidopsis. Development 126, 1109-1120 (1999).

28. Kieffer, M. \& Davies, B. Developmental programmes in floral organ formation. Semin. Cell Dev. Biol. 12, 373-380 (2001).

29. Pidkowich, M. S., Klenz, J. E. \& Haughn, G. W. The making of a flower: control of floral meristem identity in Arabidopsis. Trends Plant Sci. 4, 64-70 (1999).

30. Zhao, Z. et al. Identification of candidate genes involved in curd riceyness in cauliflower. Int. J. Mol. Sci. 21, 1999 (2020).

31. Yousef, E. A. A., Lampei, C. \& Schmid, K. J. Evaluation of cauliflower genebank accessions under organic and conventional cultivation in Southern Germany. Euphytica 201, 389-400 (2015).

32. Labate, J. A., Robertson, L. D. \& Baldo, A. M. Inflorescence identity gene alleles are poor predictors of inflorescence type in broccoli and cauliflower. J. Am. Soc. Hortic. Sci. 131, 667-673 (2006).

33. Chen, H., Nelson, R. \& Sherwood, J. Enhanced recovery of transformants of Agrobacterium tumefaciens after freeze-thaw transformation and drug selection. Biotechniques 16, 664-668, 670 (1994).

34. Sambrook, J., Fritsch, E. \& Maniatis, T. Molecular Cloning: A Laboratory Manual. (Cold Spring Harbor Press, New York, 1989).

35. Porebski, S., Bailey, L. G. \& Baum, B. R. Modification of a CTAB DNA extraction protocol for plants containing high polysaccharide and polyphenol components. Plant Mol. Biol. Rep. 15, 8-15 (1997).

36. Thompson, J. D. et al. The CLUSTAL_X windows interface: flexible strategies for multiple sequence alignment aided by quality analysis tools. Nucleic Acids Res. 25, 4876-4882 (1997).

37. Tukey, J. W. Exploratory Data Analysis (Addison-Wesley, Reading, 1977).

38. Kruskal, W. H. \& Wallis, W. A. Use of ranks in one-criterion variance analysis. J. Am. Stat. Assoc 47, 583-621 (1952). 